\title{
A Route Search System in Consideration of the Reservation Service in Amusement Parks for Smart Phone
}

\author{
Takahirio Shibuya, Katsutoshi Kanamori, and Hayato Ohwada
}

\begin{abstract}
Many amusement parks adopt a reservation service(e.g. Fast pass at Disneyland), that effectively reduce the waiting time for visitors. Even if visitors do use the reservation service, the traveling time may be long, depending on the order in which users visit the attractions. We think that people need a new route search algorithm to enhance the reservation service. Therefore, we have developed a new algorithm employingstructured programming. We constructed the system to be executed on a smart phone by using constraint logic programming and Java.
\end{abstract}

Index Terms-Traveling salesman problem, traveling problem in amusement parks, smart phone, ECLiPSe.

\section{INTRODUCTION}

There are many attractions within popular amusement parks, making it difficult for visitors to find the fastest way of moving about. A visitor choosing a very slow route may become tired from walking and waiting, and may miss the opportunity to experience the desired attraction. We think that visitors could move around more quickly if there were a system to find the fastest order.

This traveling problem is similar to the "traveling salesman problem". A traveling salesman must find the shortest possible route that visits each of a group of cities exactly once, given a list of the cities and their pair wise distances.

Research is being conducted to solve the attraction routing problem by applying the traveling salesman problem. For example, research is being conducted to determine how best to move around the 2005 World Exposition, in Aichi, Japan using a two-opt method and a simulated annealing method, which is a meta-heuristics method used to search for an approximate solution.[1] Research is also being conducted to determine how to travel efficiently using CPLEX.[2]

In this paper, we consider about two cases. The first case has no reservation service. A reservation service enables visitors to enjoy attraction without waiting. For example, Fastpassat Disneyland is a reservation service. In this case, we solve the problem by applying the traveling salesman problem as above. The other case has a reservation service. In this case, we propose a suitable route-search algorithm. Hereafter, we call this new algorithm the FP algorithm, and the traditional traveling salesman algorithm the TP algorithm.

Manuscript received September 12, 2012; revised November 7, 2012. This work was supported in Research Institute for Science and Technology in Tokyo University of Science.

The authors are with the Industrial Administration Department, Tokyo University of Science, 2641 Yamazaki, Noda, Chiba, Japan (e-mail: j7411616@ed.noda.tus.ac.jp; ohwada@ia.noda.tus.ac.jp).
In addition, we construct a system that visitors can actually use.

Our goal is thus to develop a route-search algorithm to enhance the existing reservation service and a second route search system that visitors can use on their smart phones.

The rest of the paper is organized as follows. In the next section, we describe the characteristics of this study and then give an example of its application to an actual amusement park to demonstrate the effectiveness of this method in Section 3. We verify our system in Section 4 and then we conclude in Section 5.

\section{Proposed System}

In this paper we consider an approach using the traveling salesman problem. The traveling salesman problem (TSP) is an NP-hard problem in combinatorial optimization studied in operations research and theoretical computer science. Given a list of cities and their pair wise distances, the task is to find the shortest possible route that visits each city exactly once and returns to the origin city. It is a special case of the traveling purchaser problem. In this study, we regard the cities in the traveling salesman problem as attractions in the amusement parks.

However, whereas the salesman must visit all cities in the traveling salesman problem, it is impossible for a visitor to visit all attractions in this attraction problem. Accordingly, users select the attractions they want to visit, and then we apply the traveling salesman problem method to the selected attractions.

In this paper, we use a constraint logic programming language and solve this problem using an algorithm called the branch and bound method. We call this algorithm the TP algorithm.

It is difficult to employ this algorithm with a reservation service because of the constraint whereby the usage time of the reservation service is limited. We therefore constructed the system using simple structured programming.

\section{A. Minimizing Time, Including Transit Time, Waiting Time, and Seat-Loading Time}

We determine the optimum path to minimize the total time, including transit time, waiting time and seat-loading time. We generate specific formulation to find the optimal solution. First, we define the following symbols.

We define $I$ as a set of attractions and $T$ as a set of time zones. We further define $M_{i j}$ as the transit time from "attraction $i \in I$ "to" attraction $j \in I^{\text {" }}, W_{i t}$ as the waiting time of "attraction $i \in I$ "when the time is $t \in T, P_{i}$ as the seat-load time of "attraction $i \in I$ "and $\{(i, j) \mid i, j \in I\}$ as a set of branches. The branching in this case is that of the path 
between any two attractions. $X_{i j}$ is a 0 -1variable, defined as follows.

$X_{i j}=\left\{\begin{array}{l}1 \ldots \text { when there is a path from attraction } i \in I \text { to } j \in I \\ 0 \ldots \text { when there is no path from attraction } i \in I \text { to } j \in I\end{array}\right\}$

Equation 1 determines acyclic path that minimizes the time required.

$$
\sum_{(\mathrm{i}, \mathrm{j}) \in \mathrm{A}}\left(M_{i j}+W_{i t}+P_{i}\right) X_{i j} \rightarrow \min
$$

\section{B. Minimization of Time Considering are Servation Service}

Next, we describe minimization algorithm applied to are servation service. A reservation service is a system like those used at Universal Studios or Disney land.

A reservation service's specification greatly depends on amusement parks. In this paper, we describe Disney land's Fastpass as an example.

Disney Fastpass tickets are dispensed by machines outside each attraction that uses them. The visitor inserts his/her park ticket into a reader on the machine. The machine then returns the admission ticket and prints out a Fastpass ticket. This ticket shows the time window during which the visitor may enter the special priority line at that attraction. The time period given is normally one hour for rides, and 15 minutes for theatrical presentations. It also indicates when another Fastpass can be obtained.

In normal practice, only one Fastpass ticket can be held at a time. Another Fastpass ticket can be obtained either at the start of the current Fastpass ticket's return time or after two hours, whichever is earlier. If a visitor attempts to obtain another Fastpass before this time, an informational ticket will be printed indicating when the next Fastpass ticket can be obtained.

The presence of Fastpassat and attraction does not imply that Fastpass tickets will be offered on any given day. During less-crowded operating days, the system may not be used on certain attractions as the stand-by wait is expected to be short enough to make Fastpass unnecessary. Fastpass is generally not used at all during separate-ticket events, such as Mickey's Very Merry Christmas Party, or during special after-hours events for resort visitors or annual pass holders, although exceptions do occur.

The Fastpass constraints are as follows.

1. Fast pass is only available within the time listed on the ticket.

2. Once you have a Fastpass, it is not possible to obtain until two hours from the time on the ticket.

The above constraints make it difficult to find the minimum route considering Fastpass.

In this study, we decided to use structured programming rather than constraint logic programming to solve this problem. Although structured programming takes more time, it is possible to find the minimum value.

We assume that visitors ride all of the attractions they select and use the Fastpassat one of them. We present pseudo-code below to find the minimum time for that case.

It is clear that getting a Fastpass upon arrival is most effective. In this paper, we assume that this is clear and put the activity of obtaining a Fastpass first. Thus, visitors obtain a Fastpass upon arrival and then ride to the first attractions.

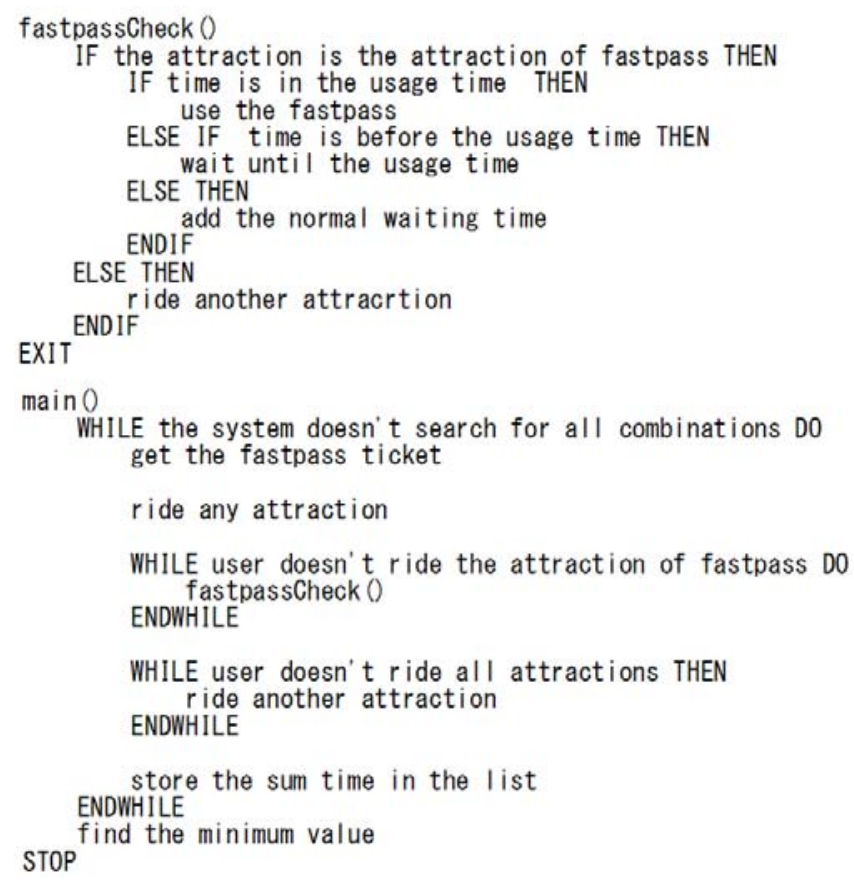

The Fastpass Check subroutine checks whether a given attraction can use Fastpass. If the attraction can use Fastpass, our system considers three situations. One is that the current time is within the Fastpass usage time. In this case, we regard the waiting time as zero. The second situation is that the current time is before the Fastpass usage time. In this case, we add as waiting time the time until the usage time. The third is that the current time is after the Fastpass usage time. In this case, we add the normal waiting time because the visitor cannot use Fastpass. After the initial check, if the attraction cannot use Fastpass, we simply add the attraction's waiting time.

After the Fastpass's attraction is visited, we add the rest of the attraction's riding time. Thus, our system seeks the total time and calculates all combinations and finds the minimum time. This method can calculate the shortest time.

Consider that the visitor has selected some Fastpass attractions. The system has to repeat the above process. For example, if Big Thunder Mountain and Splash Mountain are among the attractions that the visitor has selected, our system goes though the above process two times. The system regards $\mathrm{M}_{1}$ as the shortest time before visitor applies the Fastpass to Big Thunder Mountain and $\mathrm{M}_{2}$ as the shortest time before the visitor applies the Fastpass to Splash Mountain. In that case, the shortest time (Min) is given by the following.

$$
\operatorname{Min}=\min \left(M_{1}, M_{2}\right)
$$

\section{Applying GPS}

The Global Positioning System(GPS) is utilized in the system and in the smartphones to make this system more useful.GPSis a space-based satellite navigation system that provides location and time information in all weather conditions, anywhere on or near the Earth where there is an unobstructed line of sight to four or more GPS satellites. By adding this function, a visitorwill be able to search based on his/her current location. 
There are three requirements to apply GPS. One is to obtainthe latitude and longitude of the current location and of each attraction. Thesecan be easily obtained by using the Google Maps API.

The second requirement is to calculate the relevant distancesusing the latitude and longitude of the above-mentioned points.We use Hubeny's formula to calculate the distance.

In this study, the Earth is assumed to be a sphere with a equatorial radiusof $\mathrm{r}=6878.1 \mathrm{~km}$. We want travel time not the travel distance. We assume that the visitor moves over the shortest distancesat 3 kilometers per hour and convert these distances into travel times.

Finally, the system outputs the shortest total time required by using the travel time from the above. In this way, apath search beginning at the current location is possible.

\section{IMPLEMENTATION}

In this paper, we donot justproposea formula;we alsobuild a system that visitors can actually use. When we assume that the system is actually used,we mean that actual visitorshave to be able to use this system while at the amusementpark. Considering this, a system thatcan be manipulated on a smartphone is the most appropriate. Therefore, we built a system that is executed on a smartphone rather than on a PC or laptop.

\section{A. Example}

\section{1) Subject area}

We constructed a system for Tokyo Disneyland in Chiba Prefecture in Japan as an example. A visitor chooses eight of the thirty-one attractions that he/she would like to visit. We assume that the visitor can visit all chosen attractions, without considering that service may be suspended at one or more of them.

\section{2) How to determine the time-wait time and travel time}

The shortest distance between attractions is measured with "Kyorisoku"[10], a map service used to measure distances provided by MapionCo.,Ltd.(a Japanese company). When we measure distances, we use them to be measured on the map with Kyorisoku. We assume walking speed of three kilometers per hour and use this value for calculating the transit times.

We assume that the attraction's entrance and exit are at the center of the attraction and that the center point leads to the nearest street because we cannot determine the attraction's entrance and exit from the map.

We adopt data taken from the official site of Tokyo Disneyland[11] as the seat-loading time. Actual waiting times were published in October 2008 by Tokyo Disneyland. We also utilized the site "Congestion expectation calendar in Tokyo Disneyland"[11] to determine waiting times.

Business hours actually differ by date, but we assumed that Tokyo Disneyland is open from $8 \mathrm{AM}$ to $10 \mathrm{PM}$.

\section{B. System Outline}

We can construct a web application using the thisECLiPSe program in two ways.

One way is to interact with Java. ECLiPSe can interact with Java [13] using a Java-ECLiPSe interface. The
Java-ECLiPSe interface is a general-purpose tool for interfacing ECLiPSe with Java, Sun's popular object-oriented platform-independent programming language. For example, it could be used to write a Java graphical front-end for an ECLiPSe optimization program or to interface ECLiPSe with a commercial application written in Java.

The other way is to execute Java on the web with a Java servlet. A Java servlet is a program for dynamically generating an HTML document for a web page with Java. We construct a dynamic web site for a smart phone to use this function.

Fig. 1 illustrate the structure of our system. The system incorporates Apache and Tomcat; Apache is used to build a web server, and Tomcat is used for the Java servlet.

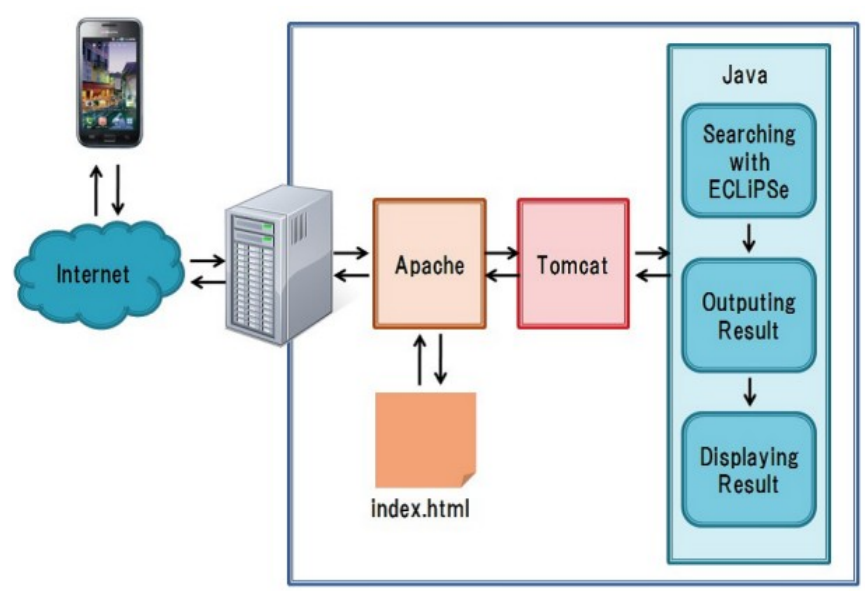

Fig. 1. System structure

Our system has the following interfaces. Fig. 2 depicts the top screen. This screen is displayed when a visitor first accesses our site. The visitor then inputs the starting time, whether it is aweekday or holiday, and the attractions lists from this screen. After two or three seconds, the result screen (Fig. 3) will display the starting time, arrival time, weekday or holiday, the total time, and the minimum route for visiting the attractions.

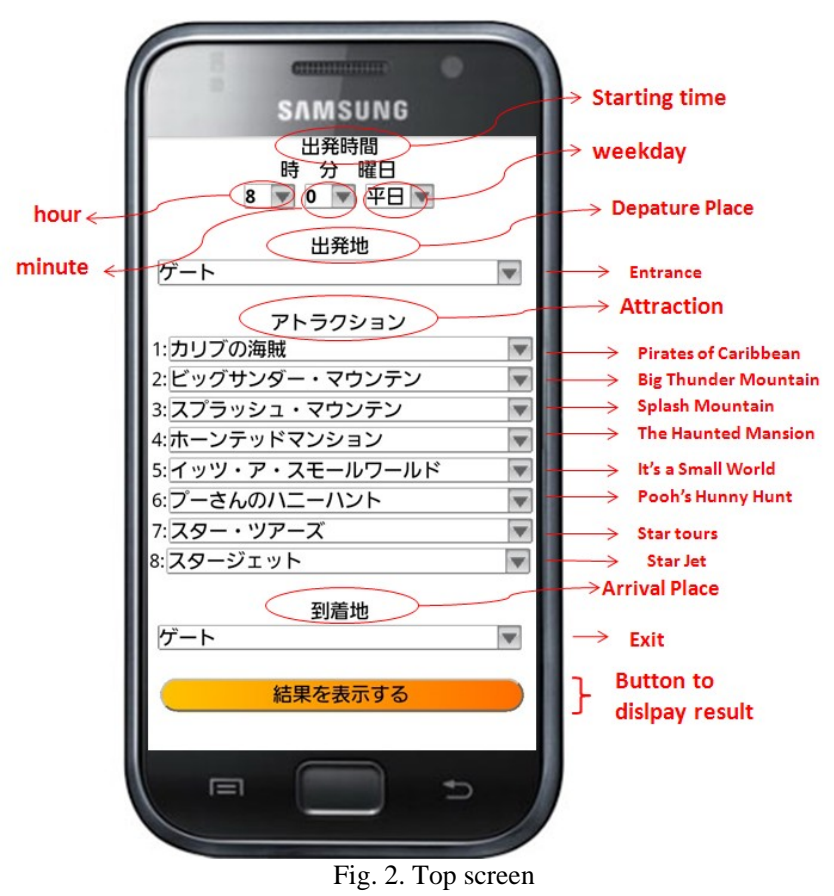




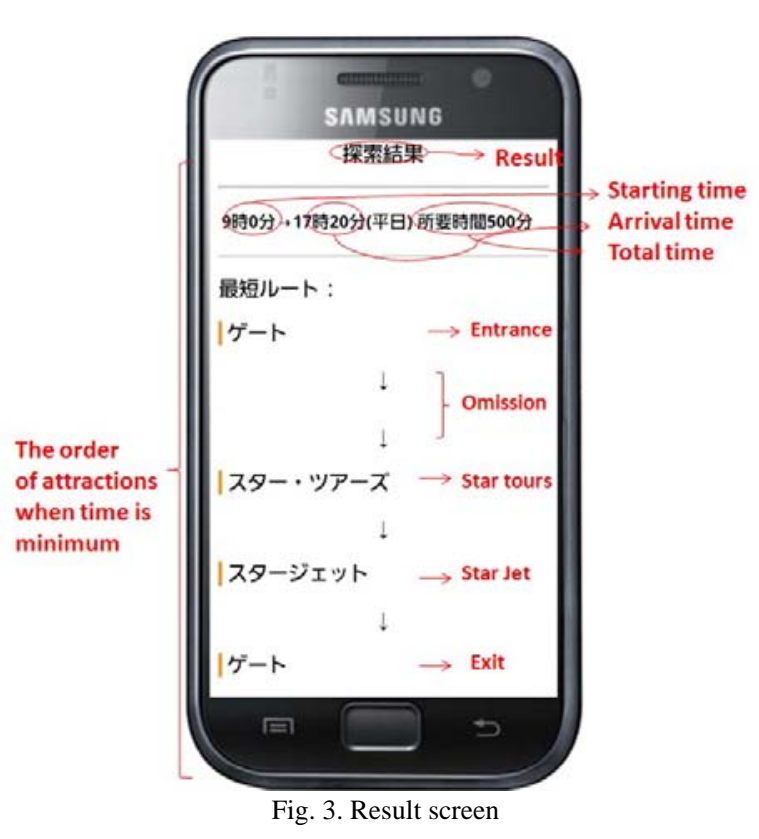

TABLE I: ATTRACTIONSAT TOKYO DISNEYLAND

\begin{tabular}{|c|c|}
\hline ID & Attraction \\
\hline 1 & Splash Mountain \\
\hline 2 & Space Mountain \\
\hline 3 & Pirates of the Caribbean \\
\hline 4 & The Haunted Mansion \\
\hline 5 & Big Thunder Mountain \\
\hline 6 & Pooh'sHunny Hunt \\
\hline 7 & Buzz Lightyear \\
\hline 8 & Star Tours \\
\hline
\end{tabular}

A. Verification Environment

Table II lists the system hardware and software.

TABLE II: HARDWARE AND SOFTWARE USED

\begin{tabular}{|c|c|}
\hline CPU & Intel(R) Celeron(R) CPU 2.00GHz \\
\hline Memory & $1.03 \mathrm{~GB}$ \\
\hline OS & CentOS 5.3 \\
\hline ECLiPSe & ECLiPSe 6.0 \\
\hline Java & Jdk 1.6.0_25 \\
\hline Tomcat & Tomcat 6.0 \\
\hline Apache & Apache 2.2.3 \\
\hline
\end{tabular}

\section{B. Results}

The results are presented in Table III. Notice that the number of attraction to travelwhen using Fastpassis one more than when not usingaFastpass,because in the former case the visitor needs to obtaina Fastpass. The resultshows that the visitor should apply theFastpass to the Hunny Hunt.The specified route is the faster route to visit the given attractions.

TABLE III: TOTAL TIME

\begin{tabular}{|c|c|c|c|c|}
\hline & run time & distance & traveling time & route \\
\hline $\begin{array}{c}\text { TP } \\
\text { Algorithm }\end{array}$ & $0.97(\mathrm{~s})$ & $3200(\mathrm{~m})$ & $735.0(\mathrm{~min})$ & $\begin{array}{c}8-2-5-3- \\
7-4-6-1\end{array}$ \\
\hline $\begin{array}{c}\text { FP } \\
\text { Algorithm }\end{array}$ & $18.63(\mathrm{~s})$ & $3775(\mathrm{~m})$ & $617.3(\mathrm{~min})$ & $\begin{array}{c}6-8-2-1- \\
6-3-7-4-5\end{array}$ \\
\hline
\end{tabular}

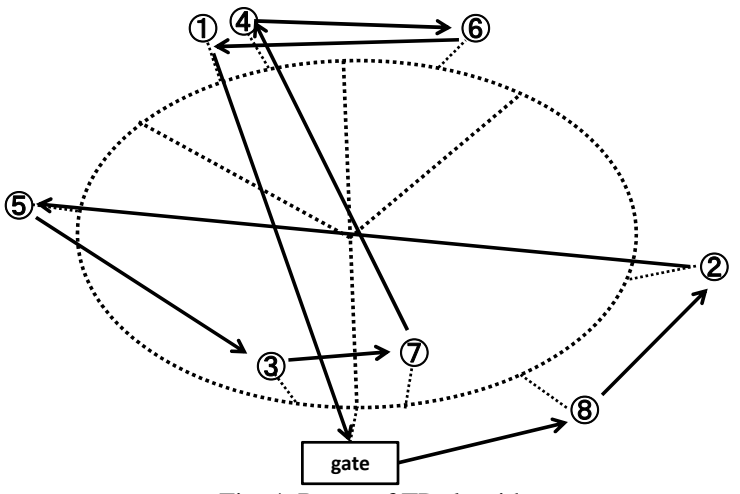

Fig. 4. Route of TP algorithm

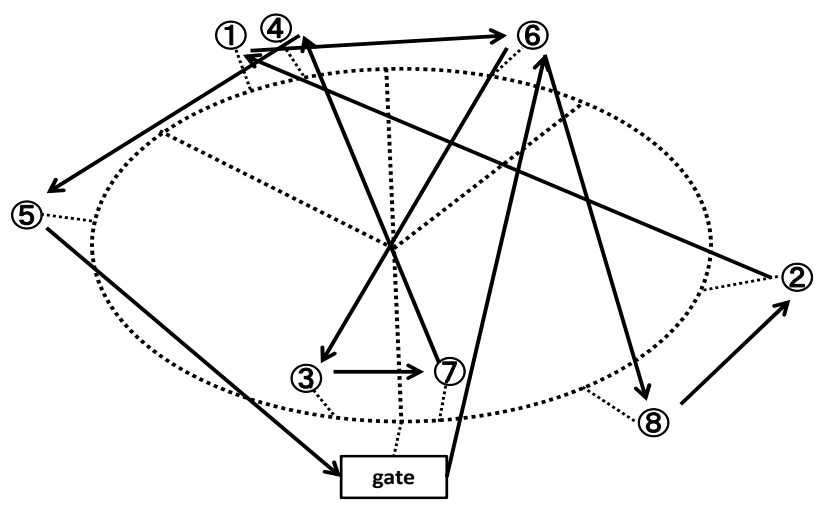

Fig. 5. Route of FP algorithm

\section{Discussion}

The FP algorithm route is 105 minutes shorter thanthe TP algorithm. However, with respect to the execution time, the FP algorithm is very much longer than the TP algorithm,because the attractions visitor selected include six attractions that useFastpass. In this example, the system repeats the process of $\Pi \mathrm{B}$ six times. Therefore, the execution time becomes longer.

In a further analysis, we compared the distance of the TP algorithm with that of the FP algorithm. Figure 6, plots the TP and FP distance for routes from the shortest to the fifth shortest in total time.

We expected that the FP algorithm distance would exceedthat of TP, but the actual results were similar. The distance of the third shortest route is comparable to the TP algorithm. In the future, we will have to consider not only the total time but also the distance.

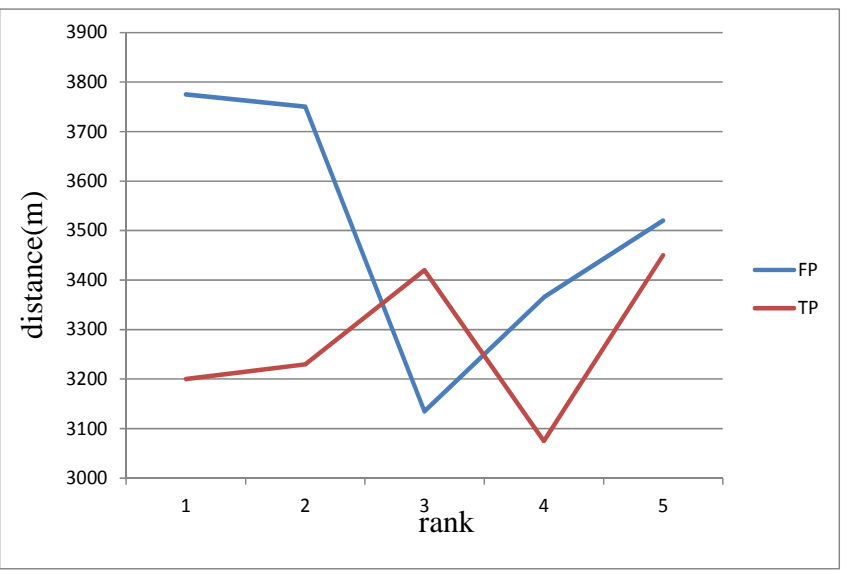

Fig. 6. TP algorithm distance versus FP algorithm distance 


\section{COCLUSION}

In this study, we constructed a system for planning routes within Tokyo Disneyland using a smart phone and presented the system outline and interface.We also compared the computation timesof theFP algorithm andthe TP algorithm. We concluded that our system is useful because it reduces the time required by about 105 minutes. However, the execution time is too long. In the future, we plan to resolve this problem using constraint logic programming.

\section{ACKNOWLEDGMENTS}

We thank the Research Institute for Science and Technology at Tokyo University of Science for financial support.

\section{REFERENCES}

[1] M. Yuki, S. Hisayoshi, T. Miho, OR of Amusementpark as an example of The 2005 World Exposition, Aichi, Japan, [Online]. Available:http://www.seto.nanzan-u.ac.jp/msie/grthesis/ms/2005/inde x.html

[2] H. Syouta, H. Yuka, and N. Daisuke, The minimumroute in universal studio Japan, [Online]. Available: http://www.seto.nanzanu.ac.jp/msie/gr-thesis/ms/2007/04mm 10.pdf

[3] S. Lin and B. W, "Kernighan An Effective Heuristic Algorithmfor the Traveling-Salesman Problem,” Operations Research, vol. 21, no. 2, pp. 498-516, 1973

[4] T. Stutzle and H. Hoos , "MAX-MIN Ant System and localsearch for the traveling salesman problem," EvolutionaryComputation, IEEE International Conference, pp. 309-314,1997.

[5] H. Takashi and K. Takayuki, and I. Tohru, "Solving vehiclerouting problems with soft time windows using chaoticneurodynamics," IEICE transactions on fundamentals ofelectronics, communications and computer sciences, vol. 105, no. 675, pp.17-22. 2006,

[6] S. Takahiro, H. Yoshihiro, and N. Koji, "Inversed FunctionDelayed Network for Traveling Salesman Problem," IEICEtransactions on fundamentals of electronics, communicationsand computer sciences vol. 107, no. 328, pp. 55-60, 2007.

[7] M. Takenori and M. Naoki, "Comparison of ApproximateMethods for Traveling Salesman Problem. IEICE transactionson fundamentals of electronics,” communications andcomputer sciences , vol. 625, pp. 1-6, 2102

[8] G. Eiichi, T. Etsuji, and W. Mitsuo, “A Randomized andGenetic Hybrid Algorithm for the Traveling Salesmanproblem. Information Processing Society of Japan,” vol. 27, pp. 61-64, 2001,

[9] Yves. Caseau and Laburthe. Francois, "Solving Small TSPswith Constraints" in Proce, the 14th International Conferenceon Logic Programming pp. 316-330, 1997.

[10] Cattafi. Massimiliano and Gavanelli. Marco, Nonato. Maddalena, Alvisi. Stefano, and Franchini. Marco, "Optimal Placementof Valves in a Water Distribution Network withCLP(FD)," The 27th International Conference on Logic Programming, pp. 731-747, 2011.

[11] Map ion, [Online]. Available: http://www.mapion.co.jp/route/

[12] Tokyo Disney resort, [Online]. Available: http://www.tokyodisneyresort.co.jp/tdl/

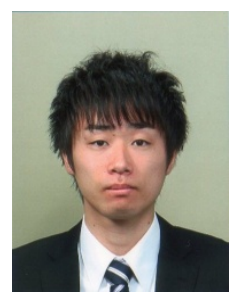

Takahiro Shibuya is Graduate Studentof Industrial Administration,Tokyo University of Science.

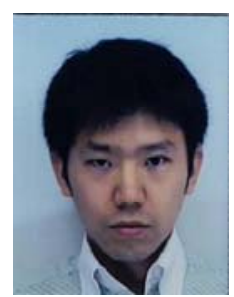

KatsutoshiKanamori is Assistant Professor of Industrial Administration, Tokyo University of Science. He received Doctor ofScience from Tokyo University of Science 2009.

He has been working on Artificial Intelligence and Formulation of Creativity.

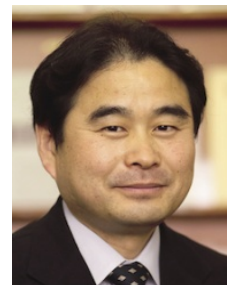

HayatoOhwada is Professor of Industrial Administration and Directorof Division of Next Generation Data Mining Technology, TokyoUniversity of Science. He received DoctorEngineering from Tokyo University of Science 1988.

He has been workingon Machine Learning and Inductive Logic Programming. 